\title{
Transfer of Vibrational Coherence Through Incoherent Energy Transfer Process in Förster Limit
}

\author{
Tomáš Mančal ${ }^{1}$, Jakub Dostál ${ }^{1,2}$, Jakub Pšenčík ${ }^{1}$, and Donatas Zigmantas ${ }^{2}$ \\ ${ }^{1}$ Faculty of Mathematics and Physics, Charles University in Prague, \\ Ke Karlovu 5, CZ-121 16 Prague 2, Czech Republic and \\ ${ }^{2}$ Department of Chemical Physics, Lund University, Getingevägen 60, 22100 Lund, Sweden
}

\begin{abstract}
We study transfer of coherent nuclear oscillations between an excitation energy donor and an acceptor in a simple dimeric electronic system coupled to an unstructured thermodynamic bath and some pronounced vibrational intramolecular mode. Our focus is on the non-linear optical response of such a system, i.e. we study both excited state energy transfer and the compensation of the so-called ground state bleach signal. The response function formalism enables us to investigate a heterodimer with monomers coupled strongly to the bath and by a weak resonance coupling to each other (Förster rate limit). Our work is motivated by recent observation of various vibrational signatures in 2D coherent spectra of energy transferring systems including large structures with a fast energy diffusion. We find that the vibrational coherence can be transferred from donor to acceptor molecules provided the transfer rate is sufficiently fast. The ground state bleach signal of the acceptor molecules does not show any oscillatory signatures, and oscillations in ground state bleaching signal of the donor prevail with the amplitude which is not decreasing with the relaxation rate.

Keywords: 2D coherent spectroscopy, vibrational coherence, coherence transfer, energy transfer
\end{abstract}

\section{INTRODUCTION}

Ultrafast time-resolved non-linear spectroscopy of electronic transitions represents an indispensable tool for the study of photoinduced dynamic and kinetic processes in wide range of interesting molecular and solid state systems [1-11]. While the degrees of freedom (DOF) of the studied systems that are directly addressed in these experiments are electronic, fine details of the time resolved spectra depend crucially on the characteristics of the surrounding nuclear modes. It was recognized early on that some ultrafast techniques yield unprecedented timedependent information about the nuclear modes, eventhough these might form a thermodynamic bath with broad spectral density. For instance, the so-called photon echo peakshift experiment yields almost directly the form of the energy gap correlation (also termed bath correlation function) [12 14]. With the advent of the two-dimensional (2D) coherent Fourier transformed spectroscopy [15, 16], it was hoped shortly that one obtained a method which would provide insight into electronic coupling between chromophores forming molecular complex. Instead, the strength of the method proved to be in allowing observation of coherent oscillatory features of electronic and nuclear origin [4, 6, 17 23]. In particular, large effort has been made to understand the vibrational features revealed by the 2D spectroscopy and to contrast them to the electronic features [22, 24 27]. Motivation for this work can be found in the ongoing debate on the origin of the long lived coherent oscillations observed in the 2D spectra of some molecular complexes [4, 66, 19, 28] for which models employing nuclear vibrational modes are also suggested [29 32]. Setting aside this particular debate, the study of the vibrational features of $2 \mathrm{D}$ spectra has its own importance. The vibrational DOF are ubiquitous in molecular aggregates and almost all ultrafast time-resolved spectroscopic methods are sensitive to them. Notable exception would be an ideal frequency integrated pump-probe spectroscopy for which one can show that it is insensitive to the nuclear vibrations [33]. 2D spectroscopy, however, can be shown to have a specific sensitivity to vibrational features [34], and the frequency resolved pump-probe spectroscopy with finite pulses keeps sufficient sensitivity to vibrational features, too.

Our theoretical work in this paper is motivated by our recent observation of coherent oscillations in low temperature 2D spectra of chlorosome 35. Previous measurements by pump-probe technique on this system showed convincingly that nuclear oscillations occur in the electronic ground state after an ultrafast excitation [36]. The chlorosome is the largest known bacterial photosynthetic antenna, containing $\sim 10^{5}$ aggregated chlorophylls which are subject to large disorder in optical gaps. Correspondingly, a plausible explanation of the oscillations observed in 2D spectra at low temperature are nuclear oscillations, as these could survive the disorder in the electronic transition energies. In our previous room temperature measurements of the chlorosome [11], we observed an initial ultrafast time scale which could be associated with the fast diffusion of the excitation between certain coherent domains formed in the disordered energetic landscape of the chlorosome. We are therefore motivated to study the influence of an ultrafast energy transfer on the evolution of the nuclear oscillatory features in the $2 \mathrm{D}$ spectra.

In order to understand the properties of nuclear oscillations observed by 2D spectroscopy of photosynthetic energy transferring systems, one needs to know spectroscopic properties and time-dependent signatures of nuclear vibrations of at least the two limiting cases in which these systems occur, namely the case of the Förster energy transfer between spatially localized excitons and the 
case of the delocalized Frenkel excitons. In photosynthetic energy transferring antennae, these cases never occur in their pure form as many photosynthetic antennae fall in between the two limiting regimes. Nevertheless, when trying to understand experimentally observed behaviors, it is useful to understand spectroscopic signatures which could be assigned these two limiting cases. In this paper, we start with the simpler of the two regimes, namely with the Förster energy transfer between spatially localized excitations. In regard to our previous work, this corresponds to the energy transfer between the coherent domains of the chlorosome [11].

To simplify the theoretical treatment, we study a weakly coupled dimeric system which possesses one pronounced intramolecular nuclear vibrational mode with sufficient Huang-Rhys factor to be observable by electronic non-linear spectroscopy, coherent 2D electronic spectroscopy in our case. We avoid the situation of a resonance between the vibrational frequency and the donoracceptor energy gap in which the selected intramolecular nuclear vibrational mode would influence the energy transfer rate. This dimer interacts with its environment (solvent, proteins surroundings etc.). We ask the question whether the vibrational coherence excited by a sequence of two short pulses (the first two of the three pulses of the 2D electronic spectroscopy technique) on one molecule (denoted as donor here) can be transferred to a neighboring molecule (an acceptor). We also study the fate of the oscillations induced on the donor after it relaxes to the electronic ground state when its excitation is transferred to the acceptor. The answers to these questions could in principle shed some light on the behavior of the coherent oscillations observed in chlorosomes.

The paper is organized as follows. In the next section we introduce the model molecular Hamiltonian and we discuss the model of system-bath interaction. We introduce the weak inter molecular coupling limit - the Förster limit. In Section $\amalg$ we derive response functions for all the signal components of the coherent $2 \mathrm{D}$ spectra of a dimer system with a weak resonance coupling, and we discuss their properties. In Section [IV we discuss particular numerical results and the dependence of the amplitude of the transferred vibrational coherence on the energy transfer rates. We present our conclusions in Section $\nabla$

\section{MOLECULAR MODEL}

In this work we consider a molecular dimer. We will describe unidirectional energy transfer from one molecule to another. We therefore denote the molecule which we consider to be the excitation donor by a letter $D$, while the other molecule (the excitation acceptor) will be denoted by $A$. In actual molecular systems, both molecules can be donors and acceptors, e.g. when they have similar transition frequency and the energy transfers in one or the other way are close to equally probable. A general theory of excitation energy transfer has to account for the possible back transfer of an excitation back to the donor molecule. However, as will be shown later, the oscillation amplitude decays during the energy transfer, and it is enough to demonstrate this on a single step of the energy transfer. In systems in which the transition frequency of the acceptor molecule will be similar to that one of the donor, such as in chlorosome, the probability of the back transfer will be diminished by the presence of other possible neighbors. Most of the relevant conclusions about the likelihood of vibrational coherence transfer can therefore be reached from studying unidirectional energy transfer. The theory developed below applies directly to a hetero dimeric system where back transfer is small due to large energy difference between the donor and acceptor.

We will describe the donor (acceptor) molecule in the dimer as a two-level system with electronic ground states $\left|g_{D}\right\rangle\left(\left|g_{A}\right\rangle\right)$ and excited state $\left|e_{D}\right\rangle\left(\left|e_{A}\right\rangle\right)$. To describe a complete non-linear spectrum of such a system, it might be necessary to add even higher lying excited states or band of states $\left|f_{D}\right\rangle\left(\left|f_{A}\right\rangle\right)$. The two molecules interact through a resonance coupling $J$ so that the electronic excitation can be exchanged between them. This means that the electronic states of the individual molecules are not eigenstates of the dimer. When, however, the interaction with the environment is stronger than the resonance coupling, the identity of the molecules is approximately preserved. Any delocalization possibly established by resonance coupling is destroyed by fluctuations induced by the environment. This is the limit of a strong bath influence, $\lambda_{\text {bath }}>J$, where $\lambda_{\text {bath }}$ is the bath reorganization energy - the parameter characterizing the systembath coupling. In the strong system-bath coupling limit, the appropriate description of the energy transfer process is the Förster rate theory and its modifications [37 39$]$. The Förster rate can be calculated from the known absorption and fluorescence spectra of the donor and acceptor molecules, respectively, and it carries a prefactor proportional to $|J|^{2}$. In this work we will be interested in the influence the value of the rate has on the transfer of the vibrational coherence. We will not calculate the rates, rather, we will use them as a free parameter assuming that different rates correspond to different values of $J$. The dynamics of the bath, which determines the line shape of the absorption and the fluorescence, will be specified in terms of the bath correlation function, also known as energy gap correlation function.

The total Hamiltonian of the dimer reads as

$$
H=H_{\text {bath }}+\sum_{i=A, D} H_{i}^{(1)}+J(|A\rangle\langle D|+| D\rangle\langle A|)+H^{(2)},
$$

where $H_{\text {bath }}$ is the Hamiltonian of the bath, $H_{i}^{(1)}$ are the Hamiltonians corresponding to the singly excited states of non-interacting dimer $H_{i}=\left(\epsilon_{i}+\Delta V_{i}\right)|i\rangle\langle i|, i=A, D$, the states $|A\rangle$ and $|D\rangle$ are the singly excited states of the non-interacting dimer

$$
|A\rangle=\left|e_{A}\right\rangle\left|g_{D}\right\rangle
$$




$$
|D\rangle=\left|g_{A}\right\rangle\left|e_{D}\right\rangle
$$

and $H^{(2)}=\left(\epsilon_{A}+\epsilon_{B}+\Delta V_{A}+\Delta V_{D}\right)\left|e_{A}\right\rangle\left|e_{D}\right\rangle\left\langle e_{D}\right|\left\langle e_{A}\right|$ is the Hamiltonian of the doubly excited state of the dimer. If needed it may include the higher excited states $\left|f_{D}\right\rangle$ and $\left|f_{A}\right\rangle$ of the donor and acceptor, respectively. The energy gap operator $\Delta V_{A}$ and $\Delta V_{B}$ describe the interaction of the acceptor and donor with the their surrounding environment, respectively. We set the ground state electronic energy $\epsilon_{g}$ to zero. We will ignore the doubly excited state in our treatment, because for weak coupling $J$ (which is assumed in our Förster type treatment) the excited state absorption cancels with the ground state contributions which would otherwise lead to crosspeaks in $2 \mathrm{D}$ spectra [40].

The description of the electron-phonon coupling (the coupling of the electronic states $|A\rangle$ and $|D\rangle$ with the bath of nuclear degrees of freedom) will be done in terms of the bath correlation functions $C_{A}(t)=\operatorname{tr}\left\{\Delta V_{A}(t) \Delta V_{A}(0) W_{e q}^{(A)}\right\}$ and $C_{D}(t)=$ $\operatorname{tr}\left\{\Delta V_{D}(t) \Delta V_{D}(0) W_{e q}^{(D)}\right\}$ which describe the fluctuation of the transition energy on the acceptor and the donor, respectively. The time argument on the energy gap operators in the definitions of the bath correlation functions denotes interaction picture with respect to the bath Hamiltonian and $W_{e q}^{(A)}\left(W_{e q}^{(D)}\right)$ denotes the equilibrium density operator of the acceptor (donor). For simplicity we will assume that their are equal, $C_{A}(t)=C_{D}(t) \equiv C(t)$, but the fluctuations on different molecules remains uncorrelated. The absorption and fluorescence spectra, as well as non-linear optical spectra including 2D spectra can be expressed using a double integral of the bath correlation function, so-called line-shape function [41]

$$
g(t)=\frac{1}{\hbar^{2}} \int_{0}^{t} \mathrm{~d} \tau \int_{0}^{\tau} \mathrm{d} \tau^{\prime} C\left(\tau^{\prime}\right) .
$$

We consider the bath correlation function, and correspondingly the line shape function, containing two components

$$
g(t)=g_{\mathrm{bath}}(t)+g_{\mathrm{vib}}(t),
$$

where $g_{\text {bath }}(t)$ describes the energy gap fluctuations due to interaction with a large harmonic bath, and $g_{\mathrm{vib}}(t)$ describes the contributions of underdamped oscillations due to individual intramolecular vibrational modes. We choose to treat the case of a single vibrational mode and we use

$$
g_{\mathrm{osc}}(t)=\frac{\lambda}{\omega}(\Theta(T)(1-\cos (\omega t))+i \sin (\omega t)-i \omega t),
$$

where $\Theta(T)=\operatorname{coth}\left(\hbar \omega / 2 k_{B} T\right)$ (see Ref. [41]). We will assume the bath to be characterized by Debye spectral density with some correlation time $\tau_{\text {bath }}$, and reorganization energy $\lambda_{\text {bath. }}$. The line shape function $g_{\text {bath }}(t)$ corresponding to this bath correlation function is linear at large values of $t>\tau_{\text {bath }}$, i.e. $g_{\text {bath }}(t) \approx \alpha t$. In particular
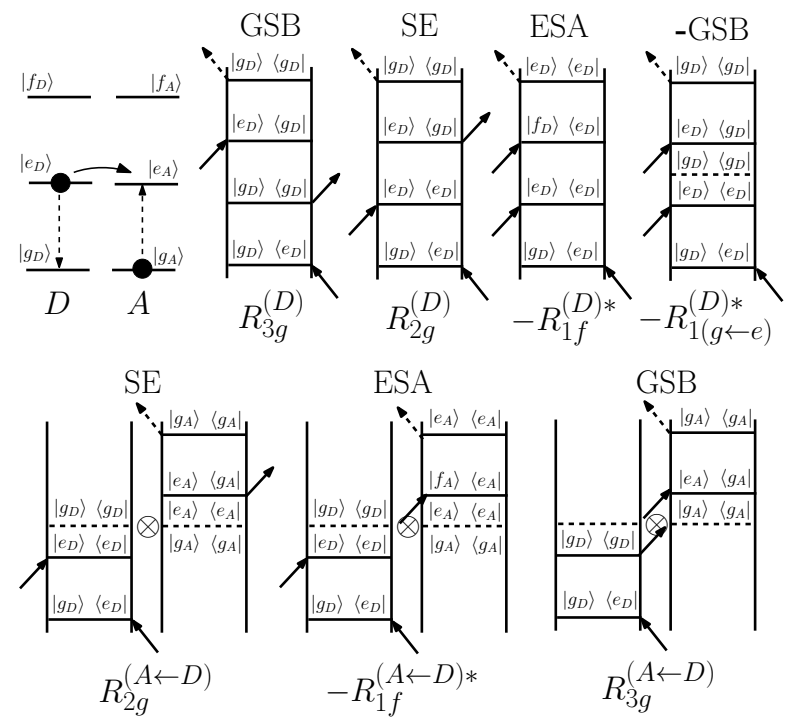

Figure 1: The diagram of the levels of a weakly coupled dimer system and the Feynman diagrams of the rephasing Liouville pathways used in this paper.

for the imaginary part we have $\operatorname{Im} g_{\text {bath }}(t) \approx-i \lambda t$ at long times [41].

The energy transfer rate $K_{A D}$ from the donor to acceptor is in principle time dependent and it can be calculated with the help of correlation functions (line-shape functions) of the donor and acceptor [42]. We assume that the part $g_{\mathrm{vib}}(t)$ of the energy gap fluctuation does not participate on the transfer rate, and we assume that the rate becomes quickly time independent. Later in this paper we study the dependence of various vibrational features in spectroscopic signals on the magnitude of the rate $K_{A D}$ which we always assume to be constant.

\section{NON-LINEAR RESPONSE FUNCTIONS WITH ENERGY TRANSFER}

Non-linear spectroscopic signals can be calculated conveniently using the response function formalism [41]. In the following, we will use the theory developed in Ref. [43] to describe non-linear optical signal of an acceptordonor system. In Ref. 43] the nuclear DOF are treated via second cumulant expansion of the response functions. For Gaussian baths, such as the bath of an infinite number of harmonic oscillators coupled linearly to the electronic transition, this approach leads to exact expressions for the response. Adding a single independent vibrational mode, which is well pronounced in the nonlinear response, allows us to model the transfer of the nuclear oscillations during energy exchange between the monomers.

In Ref. [43] two two-level systems connected through a transfer rate $K_{A D}$ were studied. In our case we need to formally include excited state absorption to higher excited state other than the two-exciton states discussed 
above. However, this addition does not change the treatment of the energy transfer, because higher excited states do not contribute to the energy transfer dynamics observed by third order spectroscopic methods (such as 2D coherent spectroscopy) [41]. We will study the transfer in one direction, from a donor molecule denoted as $D$ to an acceptor molecule denoted as $A$. We will denote nonlinear response functions corresponding to experimental signal by superindices $(D)$ and $(A)$, or $(A \leftarrow D)$ if the response contains energy transfer. We will show that this process leads to a partial loss of the amplitude of the oscillations due to vibrational coherence.

There are four types of double-sided Feynman diagrams representing four general types of response functions. These are usually denoted by lower index $n=$ $1, \ldots, 4$ in the literature (see e.g. [41]). In addition, we distinguish the pathways that include the ground state $|g\rangle$ and the excited state $|e\rangle$ only, and those involving the higher excited state $|f\rangle$. To the former we add a lower index $g$ while to the latter we add a lower index $f$. Some pathways involve relaxation from the excited state $|e\rangle$ to the ground state $|g\rangle$ and we denote them with a lower index $(g \leftarrow e)$. The list of rephasing pathways and their corresponding Feynman diagrams are presented in Fig. 11

In this section, without the loss of generality, we consider only the rephasing part of the response. All calculations presented later in Section IV will be based on a complete set of pathways. We assume that originally only the donor is excited, although in reality, the process where the role of the donor and the acceptor are exchanged occurs simultaneously. There are seven rephasing Liouville pathways with the following interpretations: (i) The ground state bleach (GSB) signal from the donor is attributed to the pathway $R_{3 g}^{(D)}$. During the population time, this contribution evolves due to the bath reorganization and the oscillation of the vibrational mode, but it does not change due to the energy transfer process. (ii) The donor stimulated emission (SE) which exponentially decays with the energy transfer rate $K_{A D}$ is described by the response function $R_{2 g}^{(D)}$ multiplied by the corresponding decay factor. (iii) When the donor decays to the ground state due to energy being transferred to the acceptor the bleach is filled with the decayed population. This process is described by the response function $R_{1(g \leftarrow e)}^{(D) *}$ where the star denotes complex conjugation. This response function has an overall type of an $R_{1}$ pathway, but it carries a minus and a complex conjugation. It rises with a factor $\left(1-e^{-K_{A D} t_{2}}\right)$. The minus leads to cancellation of the GSB contribution of $R_{3 g}^{(D)}$. (iv) Together with the energy transfer there is a corresponding rise of the GSB of the acceptor. This is described by the response function $R_{3 g}^{(A \leftarrow D)}$ and the rise is the same as in pathway (iii). (v) and (vi) Similarly to the GSB also the SE and the ESA of the acceptor rise. These processes are described by the response functions $R_{2 g}^{(A \leftarrow D)}$ and $R_{1 f}^{(A \leftarrow D)}$, respectively. (vii) Finally, the ESA of the donor is decaying in exactly the same way as its SE, and it is described by the response function $R_{1 f}^{(D) *}$ multiplied by the corresponding exponentially decaying factor.

\section{A. Transfer of the Excited State Vibrational Coherence}

Using the matrix elements of the Liouville space density matrix propagators for optical coherences $\mathcal{U}_{e g}(t) \equiv$ $\mathcal{U}_{\text {egeg }}(t)$ and the ground- and excited state density matrix elements $\mathcal{U}_{\text {ee }}(t) \equiv \mathcal{U}_{\text {eeee }}(t)$ and $\mathcal{U}_{g g}(t) \equiv \mathcal{U}_{\text {gggg }}(t)$, where we abbreviated the number of electronic indices to two, we can express the above discussed response functions in the following way (see e.g. [40]):

$$
\begin{aligned}
& R_{2 g}^{(D)} \approx\left\langle\mathcal{U}_{e_{D} g_{D}}\left(t_{3}\right) \mathcal{U}_{e_{D} e_{D}}\left(t_{2}\right) \mathcal{U}_{g e}\left(t_{1}\right)\right\rangle_{D} e^{-K_{A D} t_{2}}, \\
& R_{3 g}^{(D)} \approx\left\langle\mathcal{U}_{e_{D} g_{D}}\left(t_{3}\right) \mathcal{U}_{g_{D} g_{D}}\left(t_{2}\right) \mathcal{U}_{g_{D} e_{D}}\left(t_{1}\right)\right\rangle_{D} \\
& R_{1 f}^{(D) *} \approx-\left\langle\mathcal{U}_{f_{D} e_{D}}\left(t_{3}\right) \mathcal{U}_{e_{D} e_{D}}\left(t_{2}\right) \mathcal{U}_{g_{D} e_{D}}\left(t_{1}\right)\right\rangle_{D} e^{-K_{A D} t_{2}}, \\
& R_{1(g \leftarrow e)}^{(D) *} \approx-K_{A D} \int_{0}^{t_{2}} d \tau\left\langle\mathcal{U}_{e_{D} g_{D}}\left(t_{3}\right) \mathcal{U}_{g_{D} g_{D}}\left(t_{2}-\tau\right)\right. \\
& \left.\times \mathcal{U}_{e_{D} e_{D}}(\tau) \mathcal{U}_{g_{D} e_{D}}\left(t_{1}\right)\right\rangle_{D} e^{-K_{A D} \tau} \\
& R_{2 g}^{(A \leftarrow D)} \approx K_{A D} \int_{0}^{t_{2}} d \tau\left\langle\mathcal{U}_{e_{A} g_{A}}\left(t_{3}\right) \mathcal{U}_{e_{A} e_{A}}\left(t_{2}-\tau\right)\right\rangle_{A} \\
& \times\left\langle\mathcal{U}_{e_{D} e_{D}}(\tau) \mathcal{U}_{g_{D} e_{D}}\left(t_{1}\right)\right\rangle_{D} e^{-K_{A D} \tau}, \\
& R_{3 g}^{(A \leftarrow D)} \approx K_{A D} \int_{0}^{t_{2}} d \tau\left\langle\mathcal{U}_{e_{A} g_{A}}\left(t_{3}\right) \mathcal{U}_{g_{A} g_{A}}\left(t_{2}-\tau\right)\right\rangle_{A} \\
& \times\left\langle\mathcal{U}_{g_{D} g_{D}}(\tau) \mathcal{U}_{g_{D} e_{D}}\left(t_{1}\right)\right\rangle_{D} e^{-K_{A D} \tau}, \\
& R_{1 f}^{(A \leftarrow D) *} \approx-K_{A D} \int_{0}^{t_{2}} d \tau\left\langle\mathcal{U}_{f_{A} e_{A}}\left(t_{3}\right) \mathcal{U}_{e_{A} e_{A}}\left(t_{2}-\tau\right)\right\rangle_{A} \\
& \times\left\langle\mathcal{U}_{e_{D} e_{D}}(\tau) \mathcal{U}_{g_{D} e_{D}}\left(t_{1}\right)\right\rangle_{D} e^{-K_{A D} \tau} .
\end{aligned}
$$

Here, we omitted the prefactors containing transition dipole moments, and $\langle\ldots\rangle_{D}=\operatorname{tr}_{\text {bath }}\left\{\ldots W_{\text {eq }}^{(D)}\right\}$ correspond to the averaging over the equilibrium environmental DOF of the donor. The $\operatorname{sign}\langle\ldots\rangle_{A}=$ 
$t r_{\text {bath }}\left\{\ldots W_{\text {eq }}^{(A)}\right\}$ represents the same averaging for the acceptor. Expressions for the standard pathways of Eqs. (7), (8) and (9) are well known from the literature, see e.g. [41]. The response of Eq. (10) will be treated in more detail later. Let us first address the Eqs. (11) to (13). The averaging on the donor in these equations leads to an elimination of the dependence on the variable $\tau$ in the donor part of the response, because

$$
\begin{gathered}
\left\langle\mathcal{U}_{e_{D} e_{D}}(\tau) \mathcal{U}_{g_{D} e_{D}}\left(t_{1}\right)\right\rangle_{D}=\operatorname{tr}_{\text {bath }}\left\{\mathcal{U}_{e_{D} e_{D}}(\tau) \mathcal{U}_{g_{D} e_{D}}\left(t_{1}\right) W_{e q}\right\} \\
=\operatorname{tr}_{\text {bath }}\left\{\mathcal{U}_{g_{D} e_{D}}\left(t_{1}\right) W_{e q}\right\} .
\end{gathered}
$$

This is because the superoperator $\mathcal{U}_{e_{D} e_{D}}(\tau)$ acting on an arbitrary operator $A$ corresponds to an action of two ordinary evolution operators

$$
\mathcal{U}_{e_{D} e_{D}}(\tau) A=U_{e_{D}}(t) A U_{e_{D}}^{\dagger}(t)
$$

and the operators can be reordered in a cyclic way under the trace operation. In addition, in $R_{3 g}^{(A \leftarrow D)}$ the $\tau$ dependencecan be eliminated also on the acceptor part of the response, because

$$
\mathcal{U}_{g_{A} g_{A}}\left(t_{2}-\tau\right) W_{e q}^{(A)}=W_{e q}^{(A)}
$$

due to invariance of the equilibrium to the ground state propagation. Correspondingly we have

$$
R_{3 g}^{(A \leftarrow D)} \approx e^{-g_{A}\left(t_{3}\right)-g_{D}\left(t_{1}\right)}\left(1-e^{-K_{A D} t_{2}}\right) .
$$

In the bleach signal there is therefore no transfer of any dependency of the response on $t_{2}$. Other transfer pathways can now be expressed through the purely acceptor pathways which have a standard form, with the first time argument (from the right) equal to zero

$$
R_{2 g}^{(A \leftarrow D)} \approx e^{-g_{D}\left(t_{1}\right)} K_{A D} \int_{0}^{t_{2}} d \tau R_{2 g}^{(A)}\left(t_{3}, t_{2}-\tau, 0\right) e^{-K_{A D} \tau}
$$

$R_{1 f}^{(A \leftarrow D) *} \approx-e^{-g_{D}\left(t_{1}\right)} K_{A D} \int_{0}^{t_{2}} d \tau R_{1 f}^{(A) *}\left(t_{3}, t_{2}-\tau, 0\right) e^{-K_{A D} \tau}$.

For the stimulated emission this leads to

$$
\begin{gathered}
R_{2 g}^{(A \leftarrow D)} \approx e^{-g_{D}\left(t_{1}\right)-g_{A}^{*}\left(t_{3}\right)} \\
\times K_{A D} \int_{0}^{t_{2}} d \tau e^{2 i \operatorname{Im}\left[g_{A}\left(t_{2}-\tau\right)-g_{A}\left(t_{2}+t_{3}-\tau\right)\right]-K_{A D} \tau},
\end{gathered}
$$

which can be relatively easily evaluated. The ESA contribution depends on the particular assumptions we make about the higher excited states, and cannot therefore be evaluated without introducing further assumptions. We can consider this contribution to be similar in oscillatory features to the SE, because the source of the oscillation is the same excited state of the acceptor.

\section{B. Refilling of the Bleaching Signal}

The ground state bleach contribution to the rephasing signal, which we denote by $R_{3 g}^{(D)}$, appears stationary, and, at the first sight, unaffected by the energy transfer process. However, the transfer process is accompanied by deexcitation of the donor domain, and correspondingly, there is a signal countering the one of the $R_{3 g}^{(D)}$ pathway. Above we denoted this signal by $R_{1(g \leftarrow e)}^{(D) *}$ above. The diagram of this pathway has a form of the $R_{1}$ diagram mirror imaged, and it is used with the minus sign. According to Yang and Fleming, Ref. [43], this pathway reads

$R_{1(g \leftarrow e)}^{(D) *}\left(t_{3}, t_{2}, t_{1}\right)=R_{3 g}^{(D)}\left(t_{3}, t_{2}, t_{1}\right) e^{-2 i \operatorname{Im}\left(g_{D}\left(t_{2}+t_{3}\right)-g_{D}\left(t_{2}\right)\right)}$

$$
\times K_{A D} \int_{0}^{t_{2}} \mathrm{~d} t^{\prime} e^{-K_{A D} t^{\prime}} e^{2 i \operatorname{Im}\left(g_{D}\left(t_{2}+t_{3}-t^{\prime}\right)-g_{D}\left(t_{2}-t^{\prime}\right)\right)} .
$$

The signal canceling the GSB contains the GSB response function $R_{3 g}^{(D)}$. Later in this paper, we will evaluate all the response functions numerically. Let us however attempt a slightly more involved analysis of Eq. (21) assuming its long $t_{2}$ approximation. Because the $g_{\text {bath }}$ and the $g_{\text {osc }}$ components of the total donor line-shape function are independent, one can factorize the response function into the bath- and the vibrational parts. In addition, the bath line shape function $g_{\text {bath }}(t)$ is linear at its arguments larger than $\tau_{\text {bath }}$ and correspondingly the $t^{\prime}$ dependence on the bath part of the line-shape function vanishes. With these assumptions, we can write for $t_{2}>\tau_{\text {bath }}$

$R_{1(g \leftarrow e)}^{(D) *}\left(t_{3}, t_{2}, t_{1}\right) \approx R_{3 g}^{(D)}\left(t_{3}, t_{2}, t_{1}\right) e^{-2 i \operatorname{Im}\left(g_{\text {osc }}\left(t_{2}+t_{3}\right)-g_{\text {osc }}\left(t_{2}\right)\right)}$

$$
\times K_{A D} \int_{0}^{t_{2}} \mathrm{~d} t^{\prime} e^{-K_{A D} t^{\prime}} e^{2 i \operatorname{Im}\left(g_{\mathrm{osc}}\left(t_{2}+t_{3}-t^{\prime}\right)-g_{\mathrm{osc}}\left(t_{2}-t^{\prime}\right)\right)},
$$

i.e. the influence of the bath evolution is completely hidden in the $R_{3 g}^{(D)}\left(t_{3}, t_{2}, t_{1}\right)$ function. The integral in Eq. (22) we see only contributions originating from the intramolecular vibrations.

Integrating Eq. (22) by parts we obtain 


$$
\begin{aligned}
& R_{1(g \leftarrow e)}^{(D) *}\left(t_{3}, t_{2}, t_{1}\right) \approx R_{3 g}^{(D)}\left(t_{3}, t_{2}, t_{1}\right)\left(1-e^{-K_{A D} t_{2}} e^{-2 i \operatorname{Im}\left(g_{\mathrm{osc}}\left(t_{2}+t_{3}\right)-g_{\mathrm{osc}}\left(t_{2}\right)-g_{\mathrm{osc}}\left(t_{3}\right)\right)}\right) \\
& -2 i \frac{\lambda}{\omega} R_{3 g}^{(D)}\left(t_{3}, t_{2}, t_{1}\right) e^{-2 i \operatorname{Im}\left(g_{\mathrm{osc}}\left(t_{2}+t_{3}\right)-g_{\mathrm{osc}}\left(t_{2}\right)\right)} \\
& \times \int_{0}^{t_{2}} \mathrm{~d} t^{\prime} \omega\left[\cos \omega\left(t_{2}+t_{3}-t^{\prime}\right)-\cos \omega\left(t_{2}-t^{\prime}\right)\right] e^{-K_{A D} t^{\prime}} e^{2 i \operatorname{Im}\left(g_{\mathrm{osc}}\left(t_{2}+t_{3}-t^{\prime}\right)-g_{\mathrm{osc}}\left(t_{2}-t^{\prime}\right)\right)}
\end{aligned}
$$

Under the integral we used explicitly the form of the $g_{o s c}$, Eq. (6). At long times $t_{2}$, when $e^{-K_{A D} t_{2}} \approx 0$, the donor contribution to the overall signal consists only of the integral term

$$
\begin{gathered}
S^{(D)}\left(t_{3}, t_{2}>K_{A D}^{-1}, t_{1}\right)=R_{3 g}^{(D)}\left(t_{3}, t_{2}, t_{1}\right)-R_{1(g \leftarrow e)}^{(D) *}\left(t_{3}, t_{2}, t_{1}\right) \\
\approx-2 i \frac{\lambda}{\omega} R_{3 g}^{(D)}\left(t_{3}, t_{2}, t_{1}\right) e^{-2 i \operatorname{Im}\left(g_{\mathrm{osc}}\left(t_{2}+t_{3}\right)-g_{\mathrm{osc}}\left(t_{2}\right)\right)} \\
\times \int_{0}^{t_{2}} \mathrm{~d} t^{\prime} \omega\left[\cos \omega\left(t_{2}+t_{3}-t^{\prime}\right)-\cos \omega\left(t_{2}-t^{\prime}\right)\right] e^{-K_{A D} t^{\prime}} e^{2 i \operatorname{Im}\left(g_{\mathrm{osc}}\left(t_{2}+t_{3}-t^{\prime}\right)-g_{\mathrm{osc}}\left(t_{2}-t^{\prime}\right)\right)} .
\end{gathered}
$$

We can see that the difference between the original GSB signal and the signal coming from the filling of the GSB is proportional to the Huang-Rhys factor with a factor in a form of an integral over oscillating function. The whole factor will obviously be an oscillating function of $t_{2}$. Its numerical analysis for the range of Huang-Rhys factors between zero and one shows that its has a leading imaginary contribution. Correspondingly, the GSB signal on a donor does not vanish after the excitation leaves the donor molecule. The remaining signal is similar to the ground state contribution $R_{3 g}^{(D)}$ multiplied by $i$ and modulated by oscillating real function. The numerical results presented in Section IV confirm this conclusion.

\section{Origin of the Bleach Signal}

Interestingly, after an ideal impulsive excitation of a molecular system, no dynamic GBS (i.e. dependent on $t_{2}$ ) would arise. This can be seen directly from the form of the response function while setting $t_{1}=0$, or from an intuitive picture summarized in Fig. 2. In terms of the response function, the beach corresponds to an excitation and then deexcitation of one of the sides of the Feynman diagram (see pathway $R_{3 g}^{(D)}$ of Fig. 1). The beating which we observe in $t_{2}$ is then the beating between the static unexcited ground state wavepacket (or more precisely an equilibrium mixed state) and a perturbed wavepacket which was excited, evolved for a short time $t_{1}$ on the excited state PES, and was then transferred back to the ground state into a non-equilibrium position. If the delay $t_{1}=0$, the wavepacket does not have time to evolve on the excited state PES and is returned back to its original equilibrium state. Hence no dynamical signal arises.

During the excitation transfer, the GSB signal is compensated by an excited state population returning to the ground state. In the non-linear response corresponding to the excited states there are in principle two wavepackets (in the ket and the bra of the Feynman diagram) which were created in the excited state at two times occurring with a delay $t_{1}$. These wavepackets are projected to the ground state of the donor during the excitation transfer, and they are unlikely to have a phase as to cancel the oscillations that already occur in the ground state. It is therefore not surprising that the GSB signal will remain after the excitation is transferred. In the next section we will study the amplitude of these remaining oscillations and its dependence on the value of the energy transfer rate.

\section{DISCUSSION}

In this section, we will present results of numerical calculations of 2D coherent Fourier transformed spectra. The definition of the 2D spectrum as well as the description of the experimental technique can be found e.g. in [15, 44]. Throughout this section we use one parameters 


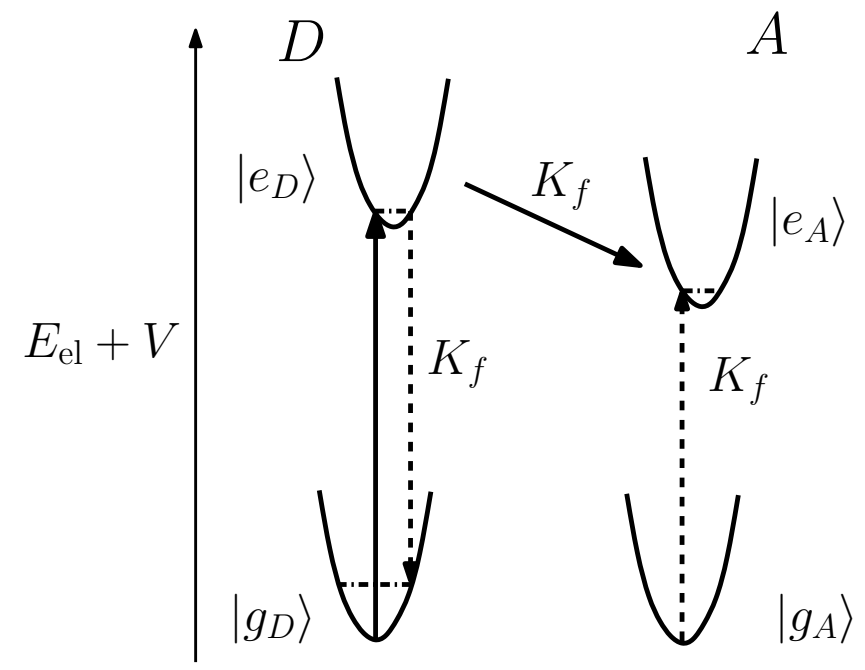

Figure 2: Potential energy surfaces of the dimer model with Förster regime of energy transfer. The donor molecule $D$ is initially excited and the excitation of is transferred with the transfer constant $K_{F}$ to the acceptor molecule $A$. This process corresponds to a deexcitation of the donor and excitation of the acceptor with the same rate $K_{F}$. The excited state wavepacket is projected to a non-equilibrium position of the ground state during the energy transfer to the acceptor.

set for the bath correlation function, namely, $\lambda_{\text {bath }}=200$ $\mathrm{cm}^{-1}, \tau_{\text {bath }}=100 \mathrm{fs}$. The temperature is assume to be $T=300 \mathrm{~K}$. These parameters ensure a realistically broad 2D spectrum on which a typical frequency of an intramolecular vibrational mode, $\omega=150 \mathrm{~cm}^{-1}$ and an oscillator reorganization frequency $\lambda<\omega$ lead to characteristic line shape modulation (see e.g. [21]). It is customary to present the real part of the spectrum, which corresponds to the absorption-absorption/stimulated emission plots [40, 44]. Similarly to the situation in chlorosome, the presence of the vibrational mode does not lead here to any discernible crosspeak, but the spectral amplitude at its center, and the overall line shape are modulated. Fig. 3 shows the characteristic oscillations of the amplitude of the 2D spectrum at the resonance, for the donor molecule with no excitation energy transfer $\left(K_{A D}=0\right)$. Fig. 4 presents the corresponding 2D spectra at some selected points (denoted by diamonds in Fig. 3). All 2D spectra are represented with respect to the resonant optical transition frequency, and they are therefore centered around the $(0,0)$ point. On both figures we can notice the initial drop of the amplitude and broadening, which occurs with the time-scale of the bath correlation time $\tau_{\text {bath }}$. For delay times longer than $\tau_{\text {bath }}$ the $2 \mathrm{D}$ spectrum shows a characteristic pattern of line shape oscillation which corresponds to an oscillation of the amplitude. The oscillations are more pronounced with increasing reorganization energy of the oscillator, i.e. with increasing Huang-Rhys factor.

When the energy transfer from the donor to the acceptor is allowed, the total donor signal is quickly disappear-

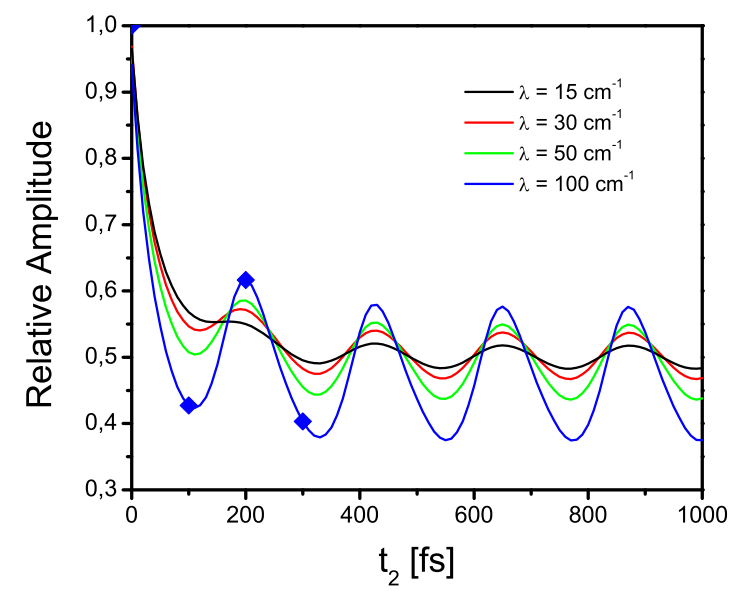

Figure 3: Oscillations due to underdamped vibrations of nuclear mode with frequency $\omega=150 \mathrm{~cm}^{-1}$ for various reorganization energies. The full blue diamonds denote the positions for which real part of the $2 \mathrm{D}$ spectrum is plotted in Fig. 4 .

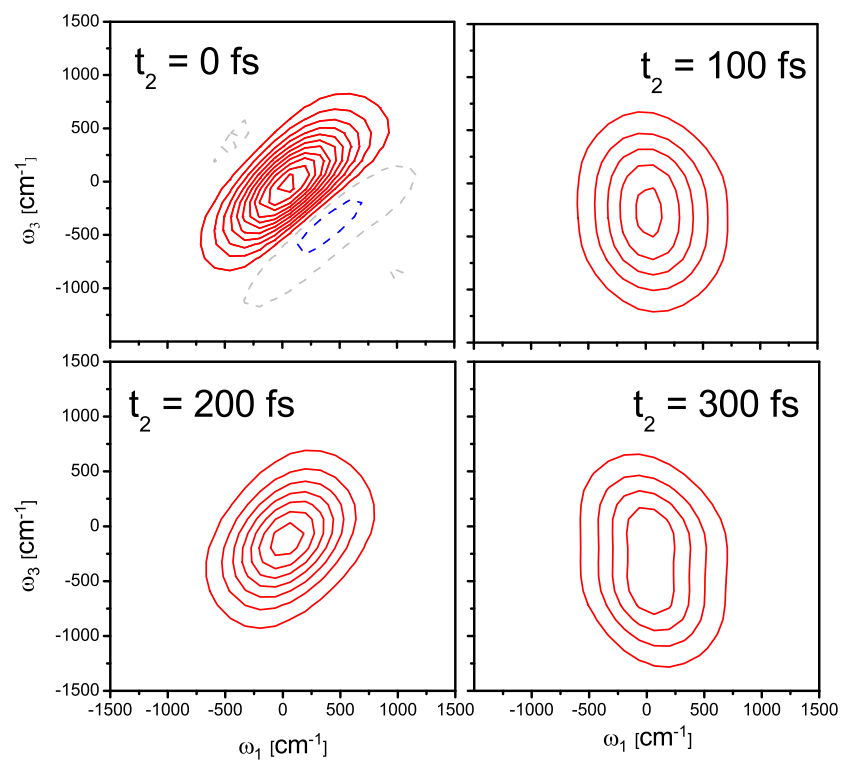

Figure 4: The time evolution of the real part of the 2D coherent spectrum of a donor molecule in the absence of energy transfer to an acceptor. The snapshots are taken at $t_{2}=0,100,200$ and $300 \mathrm{fs}$. The reorganization energy $\lambda$ of the vibrational mode is $100 \mathrm{~cm}^{-1}$. The figures correspond to the positions on a blue curve in Fig. 3 which are marked by full blue diamonds. The figures are normalized to maximum of the $2 \mathrm{D}$ spectrum at $t_{2}=0 \mathrm{fs}$, and there are 12 contours between zero and the maximum.

ing as the stimulated emission pathway decays exponentially and the ground-state bleach is compensated by the signal from the population arriving to the ground state from the excited state. Fig. 5 shows, however, that the donor signal remains oscillating around zero. The signal on the acceptor, on the other hand, rises to an amplitude 


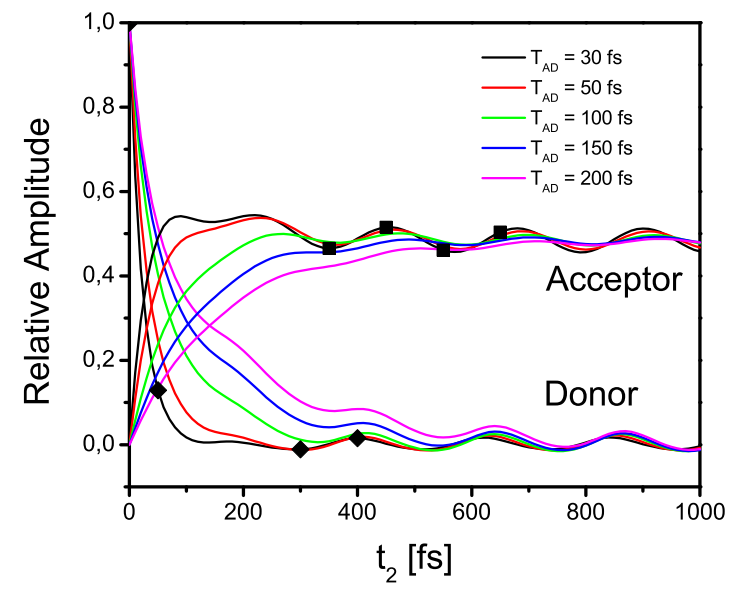

Figure 5: Oscillations of the 2D spectrum at the optical resonance. Amplitude of the spectrum at $\omega_{1}=\omega_{3}$ is plotted for the donor molecule and for the acceptor molecule for different values of the transfer time $T_{A D}=1 / K_{A D}$ and the reorganization energy $\lambda=100 \mathrm{~cm}^{-1}$. The vibrational mode has a frequency $\omega=150 \mathrm{~cm}^{-1}$. The full black diamonds indicate the positions for which the real part of the $2 \mathrm{D}$ spectrum is plotted in Fig. 6] and full back squares indicate the positions for which the real part of the $2 \mathrm{D}$ spectrum is plotted in Fig. 7

corresponding to the one exhibited by the donor without energy transfer (cf. Fig. 3). One can notice by eye that the amplitude of the oscillations decreases with the increasing transfer time (decreasing transfer rate). One can also notice that this is not the case for the ground state bleach. Characteristic 2D spectra of the donor contribution are presented in Fig. 6] Originally almost completely positive signal quickly disappears on the timescale of the energy transfer, and what remains is a signal with a characteristic shape of the imaginary part of the $2 \mathrm{D}$ spectrum (see e.g. [21]), but with an alternating sign. The rising acceptor signal, Fig. 7, shows only the characteristic line shape modulations accompanied with the change of the maximum amplitude of the spectrum.

The relative amplitude of the coherent oscillations transferred to the acceptor or left on the donor with respect to the amplitude expected on the non-transferring donor is plotted in Fig. 8, We compare relative amplitudes for four different values of the oscillator reorganization energy. We find that the transfer of oscillations to the acceptor for all reorganization energies follows roughly the same exponential decay with the transfer time. This behavior is expected, because a slow feeding rate leads to more destructive interference between various contributions of the transferred nuclear wavepacket. For electronic coherence this effect was recently discussed in Ref. [45]. Similar behavior is observed when electronic coherence is induced a multilevel molecular system by pulsed light with increasing duration as shown by Jiang and Brumer in Ref. [46]. This trend is however not fol-

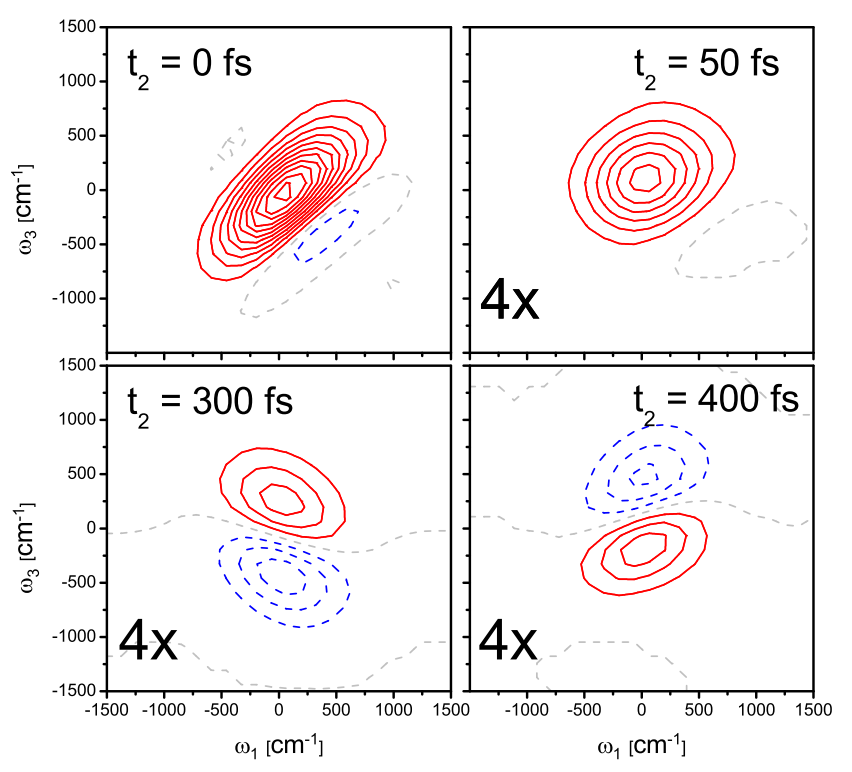

Figure 6: Decay of the signal on the donor. The short time behavior $\left(t_{2}=0\right.$ and $50 \mathrm{fs}$ ) corresponds to the bath dephasing and the loss of population. When the population is completely lost and the system has relaxed back to the groundstate electronically, the mismatch between the returning excited state nuclear wavepacket and the groudstate wavepacket yields a dispersion patern known from the imaginary part of the $2 \mathrm{D}$ spectra. The dispersion patern changes sign with the period of the oscillation. The snapshots correspond to the positions on the black curve in Fig. 5 which are marked by black diamonds. The figures are normalized to maximum of the $2 \mathrm{D}$ spectrum at $t_{2}=0 \mathrm{fs}$, and there are 12 contours between zero and the maximum. At times $t_{2}=50,300$, and $400 \mathrm{fs}$, we use four times as many contours as in the $t_{2}=0 \mathrm{fs}$ spectrum to enhance the small amplitude of the spectrum.

lowed by the GSB signal remaining on the donor. Fig. 8 demonstrates that the relative amplitude of the oscillations on the resonance does not decay with increasing transfer time. It even slightly increases at short times, and it stays flat for times up to 200 fs. For longer transfer times, the wavepacket stays for a longer time in the excited state. Because the period of the oscillator is larger than the transfer time, it apparently acquires a larger amplitude when it is projected on the ground state.

Real molecular systems are studied by the non-linear spectroscopy in form of macroscopic disordered ensembles. The chlorosome which motivates our study of the transfer of vibrational coherence is also a strongly disordered system. It is therefore worth studying the effect of the transition energy disorder on the oscillatory patter observed in $2 \mathrm{D}$ spectra. In general, a change in the transition energy results in a displacement of the 2D line shape along the diagonal line of the spectrum. The disorder thus corresponds to an additional elongation of the line shape along the diagonal line. For a line shape which is positive everywhere, this does not mean any significant change in the total amplitude of the spectrum (it should 


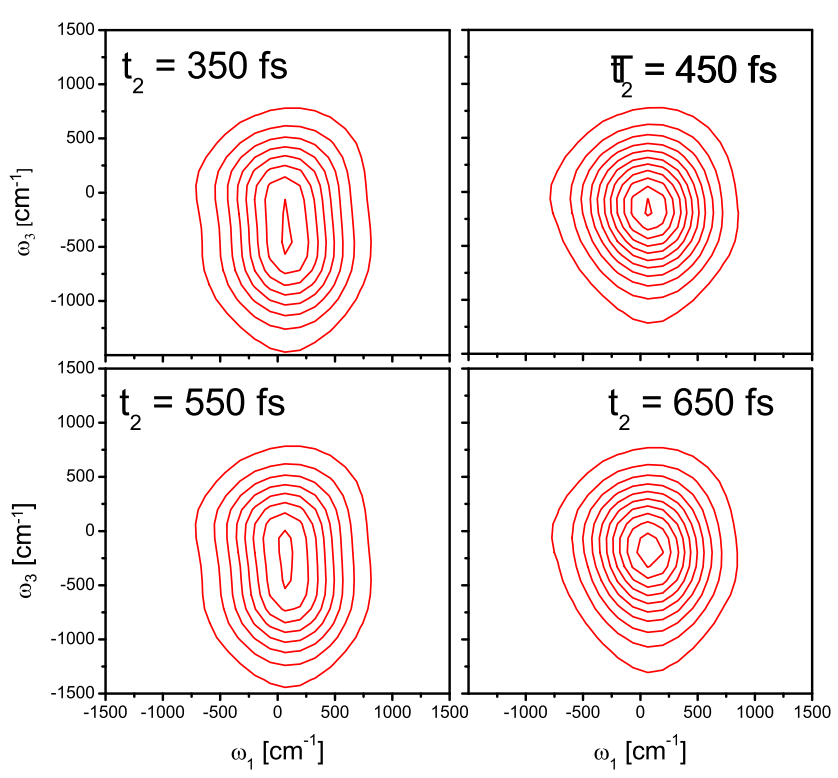

Figure 7: Time evolution of coherences transferred to the acceptor molecule. After the rise of the 2D signal is completed (see Fig. 5), the spectrum keeps evolving due the nuclear motion in the excited state of the acceptor molecule. The figures are normalized to the maximum of $2 \mathrm{D}$ spectrum at $t_{2}=450 \mathrm{fs}$ (largest amplitude of the four spectra), and there are 12 contours between zero and its maximum.

get slightly diminished due to the broadening). For a line shape corresponding to the times $t_{2}=300 \mathrm{fs}$ and $t_{2}=400$ fs in Fig. 6, however, the displacement may lead to additional canceling of the signal from differently displaced line shapes, because the line shapes contain both positive and negative regions. In Fig. 9 we therefore study the influence of the disorder on the amplitude of the transferred oscillations and on the amplitude of the remaining GSB signal. In our calculations we assume a large Gaussian disorder simulated by a normal distributions of the transition energies with a full width at half maximum of $1000 \mathrm{~cm}^{-1}$. In all studied cases the disorder leads to a decrease of the relative amplitude of the oscillations. For the oscillation transfer this decrease is less than $50 \%$. Similarly for the points of the $2 \mathrm{D}$ spectrum where the GSB has its maximum at $t_{2}=300 \mathrm{fs}$, the decay of the amplitude of the oscillations is less than $50 \%$. On the diagonal, i.e. near the nodal line of the $2 \mathrm{D}$ spectrum, the amplitude is decreased almost five times. Nevertheless, the donor signal remains oscillating despite disorder, and the expected amplitude of the oscillations in comparable to or larger than the amplitude of the transferred oscillations.

From the point of view of large aggregates the results concerning the survival of the bleach signal are the most interesting. In system where each donor has a number of acceptors, the excitation which started on a given donor will be very rarely detected returning to the donor (after it has passed through some other molecules). Even the process in which the excitation passed through its origi-

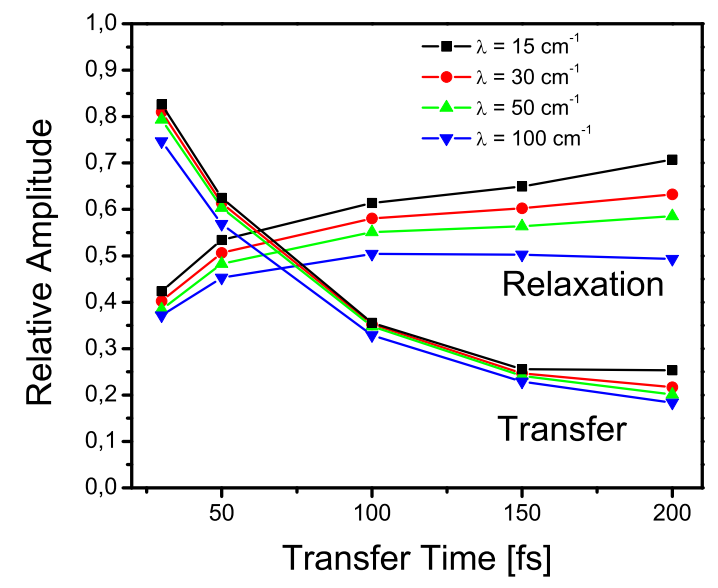

Figure 8: Efficiency of the coherence transfer. Curves doted as "Transfer": Ratio of the amplitude transferred to the acceptor to the original amplitude of the oscillations on the donor. Curves denoted as "Relaxation": Ratio of the amplitude of the ground state bleaching oscillations on the donor to the original amplitude of the oscillations on the donor. All parameters are same as in Fig. 5

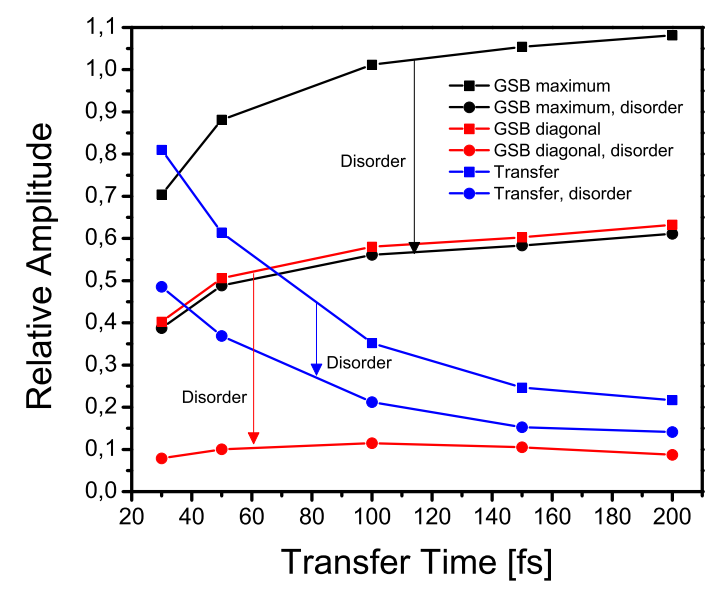

Figure 9: Influence of electronic disorder on the relative amplitude of the oscillations with frequency $\omega=150 \mathrm{~cm}^{-1}$ and reorganization energy $\lambda=30 \mathrm{~cm}^{-1}$. The disorder corresponding to a distribution of acceptor and donor transition frequencies with full width at half maximum (FWHM) equal to $1000 \mathrm{~cm}^{-1}$ was applied. The relative amplitude of the transferred coherences (in blue) is diminished only slightly. At the diagonal of the $2 \mathrm{D}$ spectru, the groud state bleaching oscillations are reduced by more than a factor of 5 (in red). However, when the amplitude is measured where the bleaching spectrum has its maximum (see lower panels of Fig. 6), the relative amplitude is decreased only by factor of 2 , and the beating on the relaxed donor remains larger than the one on the acceptor. 
nal donor and was then detected on some of the acceptors is much less frequent than the situation in which the excitation leaves the donor and never returns or passes through it. In such a case, the bleaching signal remains oscillating until the vibrational energy relaxes due to processes independent of the energy transfer process. In the same situation, continuing transfer of the excitation to subsequent acceptors will lead to a complete decay of the nuclear oscillations originating in the excited state, i.e. oscillations in the SE and ESA signals.

\section{A. Outlook}

The discussion in this paper is only a start of a more extensive research program, which has to incorporate several important effects which were neglected here. First, one has to take into account the excitonic character of the states if one wants to draw some conclusions for the possible vibrational coherence transfer in photosynthetic systems in general. Also, various transition dipole moment borrowing effects including formation of so-called vibronic (vibrational-excitonic) excitons have to be considered in case where resonance between the energy gap in the heterodimer and the vibrational frequency occurs. These two different effects amount to a study of different model situation. In the present model, however, we have also included several approximations which can be closely investigated. For instance, we assumed constant energy transfer rates, i.e. we assumed certain coarse graining of our problem in time. For fast vibrations, the relaxation rates might still be time dependent during several first periods of the vibrational motion after excitation. The relaxation rates might also be dependent on the vibrational motion itself during this initial time interval. Such effects may change the situation both quantitatively and qualitatively in some cases, and will be studied elsewhere.

\section{CONCLUSIONS}

We have investigated the stimulated emission and ground state bleach signals of a molecular dimeric system with pronounced vibrational modes. We have concentrated on the transfer of the vibrational coherence between an excited donor molecule and its neighboring acceptor. Using response function formalism adapted for the case of a resonant energy transfer with constant rates, we find that the nuclear oscillations can be transferred to a neighboring molecules. Their amplitude, however, decays with decreasing transfer rates. On the acceptor, the oscillations are solely due to the nuclear wavepacket in the electronically excited state. Interestingly, the amplitude of oscillations which prevail on the donor, after it was deexcited due to energy transfer, do not decrease with the decreasing energy transfer rate. Amplitude of both types of oscillations are decreasing in the presence of electronic disorder. In systems where excitation travels away from the original donor, the dominating and surviving contribution after many steps will be the one originating from the electronic ground state of the donor molecule.

\section{Acknowledgments}

This work was funded by the Czech Science Foundation (GACR) grant no. 205/10/0989.
[1] F. Milota, J. Sperling, A. Nemeth, T. Mančal, and H. F. Kauffmann, Acc. Chem. Res. 42, 1364 (2009).

[2] Y.-C. Cheng and G. R. Fleming, Annu. Rev. Phys. Chem. 60, 241 (2009).

[3] N. S. Ginsberg, Y.-C. Cheng, and G. R. Fleming, Acc. Chem. Res. 42, 1352 (2009).

[4] E. Collini and G. D. Scholes, Science 323, 369 (2009).

[5] D. B. Turner and K. A. Nelson, Nature 466, 1089 (2010).

[6] E. Collini, C. Y. Wong, K. E. Wilk, P. M. G. Curmi, P. Brumer, and G. D. Scholes, Nature 463, 644 (2010).

[7] G. S. Schlau-Cohen, A. Ishizaki, and G. R. Fleming, Chem. Phys. 386, 1 (2011).

[8] C. Y. Wong, R. M. Alvey, D. B. Turner, K. E. Wilk, D. A. Bryant, P. M. G. Curmi, R. J. Silbey, and G. D. Scholes, Nat. Chem. 4, 396 (2012).

[9] D. B. Turner, Y. Hassan, and G. D. Scholes, Nano Lett. 12, 880 (2012).

[10] O. Bixner, V. Lukeš, T. Mančal, J. Hauer, F. Milota, M. Fischer, I. Pugliesi, M. Bradler, W. Schmidt, E. Riedle, H. F. Kauffmann, and N. Christensson, J. Chem. Phys. 136, 204503 (2012).
[11] J. Dostál, T. Mančal, R. Augulis, F. Vácha, J. Pšenčík, and D. Zigmantas, J. Am. Chem. Soc. 134, 11611 (2012).

[12] M. Cho, J.-Y. Yu, T. Joo, Y. Nagasawa, S. A. Passino, and G. R. Fleming, J. Phys. Chem. 100, 11944 (1996).

[13] M. Yang and G. R. Fleming, J. Chem. Phys. 110, 2983 (1999).

[14] T. Mančal and G. R. Fleming, J. Chem. Phys. 121, 10556 (2004).

[15] T. Brixner, T. Mančal, I. V. Stiopkin, and G. R. Fleming, J. Chem. Phys. 121, 4221 (2004).

[16] T. Brixner, J. Stenger, H. M. Vaswani, M. Cho, R. E. Blankenship, and G. R. Fleming, Nature 434, 625 (2005).

[17] P. Kjellberg, B. Bruggemann, and T. Pullerits, Physical Review B 74, 024303 (2006).

[18] A. V. Pisliakov, T. Mančal, and G. R. Fleming, J. Chem. Phys. 124, 234505 (2006).

[19] G. S. Engel, T. R. Calhoun, E. L. Read, T.-K. Ahn, T. Mančal, Y.-C. Cheng, R. E. Blankenship, and G. R. Fleming, Nature 446, 782 (2007).

[20] D. Egorova, Chemical Physics 347, 166 (2008), cited By (since 1996): 8. 
[21] A. Nemeth, F. Milota, T. Mančal, V. Lukeš, H. F. Kauffmann, and J. Sperling, Chem. Phys. Lett. 459, 94 (2008).

[22] D. B. Turner, K. E. Wilk, P. M. G. Curmi, and G. D. Scholes, J. Phys. Chem. Lett. 2, 1904 (2011).

[23] J. R. Caram, N. H. C. Lewis, A. F. Fidler, and G. S. Engel, J. Chem. Phys. 136, 104505 (2012).

[24] T. Mancal, O. Bixner, N. Christensson, J. Hauer, F. Milota, A. Nemeth, J. Sperling, and H. Kauffmann, Procedia Chemistry 3, 105 (2011).

[25] V. Butkus, D. Zigmantas, L. Valkunas, and D. Abramavičius, Chem. Phys. Lett. 515, 40 (2012).

[26] V. Butkus, L. Valkunas, and D. Abramavičius, ArXiv 1205.3383v2 (2012).

[27] J. Seibt and T. Pullerits, J. Phys. Chem. C 117, 18728 (2013).

[28] G. Panitchayangkoon, D. Hayes, K. A. Fransted, J. R. Caram, E. Harel, J. Wen, R. E. Blankenship, and G. S. Engel, Proc. Natl. Acad. Sci. U. S. A. 107, 12766 (2010).

[29] N. Christensson, H. F. Kauffmann, T. Pullerits, and T. Mančal, J. Phys. Chem. B 116, 7449 (2012).

[30] V. Tiwari, W. K. Peters, and D. M. Jonas, Proc. Natl. Acad. Sci. U. S. A. 110, 1203 (2013).

[31] A. W. Chin, J. Prior, R. Rosenbach, F. Caycedo-Soler, S. F. Huelga, and M. B. Plenio, Nature Physics 9, 113 (2013).

[32] A. Chenu, N. Christensson, H. F. Kauffmann, and T. Mančal, Scientific Reports 3, 2029 (2013).

[33] J. Yuen-Zhou, J. J. Krich, and A. Aspuru-Guzik, J.
Chem. Phys. 136, 234501 (2012).

[34] T. Mančal, N. Christensson, V. Lukés, F. Milota, O. Bixner, H. F. Kauffmann, and J. Hauer, J. Phys. Chem. Lett. 3, 1497 (2012).

[35] J. Dostál, T. Mančal, F. Vácha, J. Pšenčík, and D. Zigmantas, submitted (2013).

[36] Y. Z. Ma, J. Aschenbrücker, M. Miller, and T. Gillbro, Chem. Phys. Lett. 300, 465 (1999).

[37] T. Förster, Annalen der Physik 2, 55 (1948).

[38] H. Sumi, J. Phys. Chem. B 103, 252 (1999).

[39] S. Jang, M. D. Newton, and R. J. Silbey, Phys. Rev. Lett. 92, 218301 (2004).

[40] T. Mančal, in Quantum Effects in Biology, edited by M. Mohseni, Y. Omar, G. S. Engel, and M. B. Plenio (Cambridge University Press, Cambridge, 2013), Chap. Principles of Multi-Dimensional Electronic Spectroscopy.

[41] S. Mukamel, Principles of nonlinear spectroscopy (Oxford University Press, Oxford, 1995).

[42] S. Mukamel and V. Rupasov, Chem. Phys. Lett. 242, 17 (1995).

[43] M. Yang and G. R. Fleming, J. Chem. Phys. 111, 27 (1999).

[44] D. M. Jonas, Annu. Rev. Phys. Chem. 54, 425 (2003).

[45] A. Chenu, P. Maly, and T. Mančal, arXiv:1306.1693 (2013).

[46] X.-P. Jiang and P. Brumer, J. Chem. Phys. 94, 5833 (1991). 